\title{
Liquen Rojo Plano Erosivo Bucal (Un Caso Clínico).
}

\section{María Silvia Almirón \\ Prof. Adjunta Cátedra Anatomía Patológica}

Andrés Benetti

Docente Adscripto Cátedra Anatomía Patológica

María Susana Briend Prof. Titular Cátedra Anatomía Patológica

José Hidalgo Especialista en Estomatologia

\section{Resumen}

El presente trabajo tiene por finalidad comunicar un caso clínico de un liquen rojo plano erosivo bucal. El liquen rojo plano oral (LPO) es una enfermedad mucocutánea crónica, de carácter inflamatorio, etiología desconocida y naturaleza generalmente autoinmune, descripta inicialmente por Wilson en 1869 quien caracterizó sus principales rasgos clínicos, y en 1906 Dubreuilh lo hizo desde el punto de vista histopatológico. Se postula un mecanismo inmunológico en la que participan los linfocitos $T$ que dañan a las células basales del epitelio luego de recibir la presentación antigénica de las células de Langerhans. Clínicamente se manifiesta como formas reticulares o atrófico erosivas y potencialmente pueden afectar a la piel y mucosas, siendo frecuente que las lesiones bucales generalmente preceden a las cutáneas o pueden presentar una sola localización intraoral. El diagnóstico debe basarse en la observación clínica y confirmarse con la descripción de las características histopatológicas del epitelio. Se presenta el caso clínico de una paciente de sexo femenino de 50 años de edad que acudió a la consulta odontológica en un Centro de Atención Primaria de la Salud en la ciudad de Corrientes por presentar descamaciones ubicadas en encía vestibular zona antero superior $y$ en mucosa yugal sector posterior con varios meses de evolución, sintomática. La paciente presentaba episodios de estrés constante en los últimos meses. Se le realizó una citología exfoliativa en primera instancia y pos- 
teriormente una biopsia incisional, las muestras antes mencionadas fueron enviadas al Servicio de Anatomía Patológica de la Facultad de Odontología de Universidad Nacional del Nordeste, donde se confirmó el diagnóstico de liquen rojo plano erosivo.

\section{Palabras claves}

Lesión blanca- Lesión precancerosa- mucosa bucal.

\section{Abstract}

The aims of this study are to communicate a clinical case of an erosive oral Lichen Planus. The Red Lichen Planus oral (LPO) is a Chronic Mucocutaneous, of inflammatory character, unknown etiology and nature usually autoimmune disease, Initially described by Wilson in 1869 who characterized his main clinical features, and in 1906 Dubreuilh did from the histological point of view. It is postulated an immunologic mechanism involving $T$ cells that damage the basal cells of the epithelium after receiving the antigenic presentation of Langerhans cells. Clinically manifested as forms reticular or atrophic-erosive and potentially can affect the skin and mucous membranes, being frequent oral lesions usually precede the skin or may present a single intraoral location. Diagnosis should be based on clinical observation and confirmed by the description of the histopathologic features of the epithelium. It is the clinical case of 50 years old female attended the dental consultation at a centre of primary health care in the city of Corrientes by peeling located in upper anterior area vestibular gingiva and buccal mucosa sector with several months of evolution, symptomatic. The patient presented episodes of stress constant in the last months. Was it an exfoliative cytology in the first instance and subsequently an incisional biopsy, the above samples were sent to the service of pathological anatomy of the Faculty of Dentistry of Universidad Nacional del Nordeste, where it was confirmed the diagnosis of Red Lichen Planus erosive.

\section{Key words}

White lesion - Precancerous Lesion - Oral mucosa.

\section{Resumo}

O trabalho presente tem para pretenda comunicar um caso clínico de um líquen plano erosivo vermelho bucal. $O$ líquen plano vermelho oral (LPO) é uma doença mucocutánea crônico, de caráter inflamatório, etiologia desconhecido e natureza geralmente autoinmune, descripta inicialmente para Wilson em 1869 quem caracterizou as características principais clínicas deles/delas e em 1906 Dubreuilh ele/ ela fez isto do histopatológico de ponto de vista. Um mecanismo imunológico é postulado no um que você / eles participa o linfocitos $T$ que danifica às celas basais do epitélio depois de receber $o$ antigénica de apresentação das celas de Langerhans. Clinicamente é manifestado goste de formas reticulares ou atrófico erosivo e potencialmente eles podem afetar à pele e mucoso, sendo freqüente que as lesões bucais geralmente precedem ao cutâneo ou eles podem apresentar um único intraoral de localização. A diagnose deveria estar baseado na observação clínica e ser confirmado com a descrição do histopatológico característico do epitélio. Ele/ela espetáculos para cima o caso clínico de um paciente de sexo feminino 50 anos velho que ele/ela entrou para a odontológica de consulta em um Centro de Atenção Primária da Saúde na cidade de Corrientes apresentar descamaciones localizado em goma área vestibular antero- superior e em yugal mucoso setor posterior com vários meses de evolução, sintomático.O paciente apresentou episódios de estrés constante nos últimos meses. Ele/ela foi levado fora um exfoliativa de citologia em primeiro exemplo e mais tarde um incisional de biópsia, as amostras mencionadas foram as correspondentes para o Serviço de Anatomia Patológica da Habilidade de Odontologia de Universidade Nacional do Nordeste onde você confirmou a diagnose de líquen plano vermelho erosivo.

\section{Palavras chaves}

Lesão branca - precancerosa de Lesão - mucoso bucal. 


\section{Introducción}

El liquen plano (LP) representa una enfermedad mucocutánea crónica de etiología desconocida, y constituye una respuesta inmunológica intermediada por células en respuesta a variaciones antigénicas que ocurren en el epitelio de la piel y de las mucosas.Las lesiones bucales de LP son muy frecuentes y pueden acompañar, anteceder o ser secundarias a las manifestaciones cutáneas. Se menciona que entre el 20 a $25 \%$ de los casos, la mucosa bucal representa la localización exclusiva del LP. Por otra parte se lo considera como una alteración en el que existe un mayor riesgo de transformación maligna. Las lesiones de liquen plano oral (LPO) exhiben una diversidad de formas clínicas, pudiendo mostrar variaciones con relación a la sintomatología dolorosa. De acuerdo con Andreasen, son conocidas seis formas clínicas en la cavidad oral: reticular, en placa (hipertrófica), atrófica, erosiva, papilar y ampollosa'.Existen lesiones bucales asociadas a liquen plano cutáneo o que a menudo aparecen solas. Usualmente afecta la mucosa bucal, labios, zona gingival, suelo de la boca y paladar (en orden descendente de frecuencia). Típicamente, las lesiones bucales constituyen líneas filiformes aterciopeladas de color blanco o gris, que poseen un patrón reticular, en cuyas intersecciones puede haber unos puntos o rayas elevados de color blanco (Estrías de Wickham).

Todos estos signos pueden encontrarse en un mismo paciente en una localización o en otra, multicéntricas, bilaterales y en un momento de la evolución o en otro. Es por ello que las posibilidades de presentación clínica son infinitas². El mecanismo del daño se iniciaría con el procesamiento de los antígenos ya sea de origen endógenos o exógenos por las células de Langerghans, quienes presentan los determinantes antigénicos a los linfocitos $T$ quienes acuden en respuesta a la interleucina'. Paralelamente se producen otros fenómenos, los linfocitos activados secretan gama interferon que induce a los queratinocitos a expresar antígenos de histocompatibilidad de clase II (HLA-DR) esto inducen un aumento en su velocidad de diferenciación y son responsables de la hiperqueratosis. Se postula que el contacto de los linfocitos y las células epiteliales induciría a transferir informa- ción antigénica incorrecta, por este mecanismo las células epiteliales reconocen autoantígeno como antígenos extraños produciendo una reacción de tipo autoinmunitario. Otros sugieren que el evento inicial es una reacción no específica que induciría a los queratinocitos a producir citocinas proinflamatorias y moléculas de adhesión que induciría a la migración de linfocitos al área epitelial subsecuente ${ }^{3}$.

Sería posible afirmar que los mecanismos moleculares que controlan el crecimiento, proliferación, maduración y apoptosis en las células epiteliales atacadas pueden jugar un papel importante en el proceso de transformación maligna ${ }^{4}$. Se la ha asociado también a infecciones virales (hepatitis $C$ ) medicaciones, alérgenos de contacto, neoplasias y stress. En la anamnesis se deben indagar por los antecedentes familiares $y$ personales $y$ en especial si toma algún fármaco o si tiene hábitos como consumo de tabaco o alcohol así como por su estado psicológico (episodios de stress) ${ }^{5}$.

Resulta que uno de los aspectos más importantes es el referido a su posible carácter premaligno. Se han reportado cifras diversas y a menudo contradictorias sobre la transformación maligna del liquen plano bucal, que van desde un 0,3 hasta un $10 \%$. Se plantea que las células epiteliales en el liquen plano bucal incrementan su tasa de proliferación en respuesta al ataque previamente descrito. Las formas clínicas erosivas se reportan como las más asociadas a la aparición de lesiones epiteliales malignas, pero los reportes en cuanto a los porcentajes de malignización son muy disímiles ${ }^{6}$. Este aparente incremento del riesgo de que aparezca un Carcinoma Oral de Células escamosas en pacientes con LPO, respecto a las personas que no lo padecen parece ser para algunos autores independiente del tipo clínico y de la terapia administrada. Además, no parece que los factores de riesgo implicados en el cáncer oral, como son el tabaco y el alcohol, jueguen un papel en la transformación del $\mathrm{LPO}^{7}$. La variante atrófica-erosiva se caracteriza por un adelgazamiento del espesor del epitelio con áreas de pérdida completa de crestas epiteliales y un denso infiltrado de linfocitos $T$ que oculta la membrana basal, manifestándose por un enrojecimiento generalizado y difuso que se extiende hasta las capas medias y 
superiores del epitelio. En la mayoría de las áreas se observa licuefacción de la membrana basal y vacuolización y destrucción de las células basales subyacentes del corion. La localización más frecuente es la lengua siendo también la encía un lugar habitual de asentamiento presentándose como una gingivitis descamativa crónica. Esta forma se caracteriza por la presencia de ulceraciones del epitelio sobre una mucosa atrófica, aunque también podría presentarse como forma ampollar ${ }^{(4)}$ Uno de los elementos morfológicos más importantes es la presencia de la displasia epitelial, como un elemento predictor de su posible desarrollo neoplásico, aspecto éste en el cual se discute mucho por los investigadores de esta enfermedad. Actualmente, el concepto de displasia epitelial es global y señala la existencia de una combinación variable de fenómenos microscópicos indicativos de un desorden de la maduración epitelial y de una alteración de la proliferación celular ${ }^{(6)}$. Al igual que sucede con su posible malignización, los porcentajes de presencia de displasia epitelial en el liquen plano bucal son muy variables, tales como un $25 \%,{ }^{2}$ I I $\%,{ }^{3} 34 \%,{ }^{4}$ II,4 \%y un $57,5 \%$. Existen evidencias para soportar la hipótesis de que mientras más severa es la displasia, mayor es la predisposición a la transformación maligna ${ }^{4}$. El diagnóstico diferencial de las lesiones de liquen plano debe realizarse, con las formas de predominio blanco, con otras lesiones blancas entre las que destacamos: lesiones por agentes mecánicos, físicos químicos; radiaciones, lesiones por agentes microbianos: sífilis, hongos, desórdenes inmunológicos, penfigoide cicatricial, lupus, hiperplasias y tumores benignos, nevo blanco, psoriasis, leucoplasias. Cuando nos hallamos ante una forma de liquen plano de predominio rojo con lesiones eritemato-descamativas, el diagnóstico diferencial debe realizarse principalmente con otras lesiones vesiculo-erosivas como el eritema multiforme, el pénfigo vulgar o el penfigoide 5 .

La indicación del tratamiento del liquen plano oral (LPO) se plantea una vez que se haya confirmado el diagnóstico de la enfermedad mediante la aplicación de criterios clínicos y estudios histopatológicos; y si fueran necesarios también inmunológicos El enfoque terapéutico se realizará en función de los siguientes parámetros: la forma clínica; los síntomas; la localización y extensión del LPO; así como el fracaso de previos tratamientos Básicamente los objetivos del tratamiento del LPO serán: la eliminación de las lesiones atróficas y erosivas, controlar los síntomas y disminuir el potencial maligno de la lesión. El primer paso a tener en cuenta es la eliminación de factores locales que pudieran exacerbar la lesión para, a continuación, pautar el tratamiento específico del LPO. Es necesario eliminar los factores que exacerban la lesión como son los hábitos orales, como también minimizar el riesgo de desencadenar el fenómeno de Koebner, evitando hábitos como el mordisqueo labial, yugal o lingual. Además si es necesario, se pulirán las restauraciones desbordantes y la eliminación de las superficies anfractuosas dentarias, y se planteará la reparación o sustitución de las prótesis en mal estado. Controlar la placa dental ya que puede producir el fenómeno de Koebner Holmstrup y cols, pusieron de manifiesto la mejoría de los pacientes con LPO gingival cuando se les instruía en un programa de higiene oral mantenido durante un año. Se ha documentado que en los pacientes con liquen plano de localización gingival, sometidos a tratamientos gíngivo-periodontales (tartrectomías y raspados gingivales) periódicos, una o dos veces al año resultan beneficiosos para su evolución. También resulta de utilidad, realizar enjuagues con clorhexidina al 0,2 \% (sin alcohol) dos veces al día como también el control del estrés o ansiedad en algunos pacientes mejora el cuadro. Debe aconsejarse a los pacientes mantener una dieta equilibrada y evitar la ingesta de alimentos que desencadenen dolor - exacerben las lesiones atrófico-erosivas (8) Presentación del Caso Clínico.

Se presenta a la consulta odontológica en un Centro de Atención Primaria de la Salud en la Ciudad de Corrientes una paciente de sexo femenino de 50 años de edad. Se le realiza la correspondiente historia Clínica médica y odontológica donde la paciente relata que las lesiones presentan una evolución de aproximadamente seis meses con sintomatología de ardor y dolor bucal. A la exploración clínica se observan descamaciones ubicadas en encía vestibular, tanto libre como adherida con presencia de placa bacteriana a nivel cervical. La encía vestibular presentaba un color rojo subido en la zona anterior 
del maxilar superior; $y$ en la zona del trígono retromolar, se observaron lesiones leucoplasiformes, como así también pequeñas lesiones nodulares blanquecinas en bordes laterales de la lengua.Se le realizó exploración cervicofacial completa no encontrándose adenopatías palpablesa nivel de las cadenas submaxilares y sublinguales. La paciente refiere dichas lesiones desde hace 6 meses manejando niveles elevados de stress en los últimos meses por razones familiares. La paciente no fuma, no consume alcohol y no presenta alergias medicamentosas, ni alimenticias conocidas. Se le explico a la paciente los pasos a seguir y con el consentimiento informado se procedió a realizar en una primera instancia una citología exfoliativa con cytobrush fijándose con alcohol de $96^{\circ}$ y se remitió al Servicio de Anatomía Patológica de la Facultad de Odontología donde se realizó la técnica de Papanicolaou para su coloración Luego se procedió a realizar una biopsia de tipo incisional realizando anestesia local y utilizando como instrumental de toma punch de tamaño mediano y grande, incidiendo de manera profunda hasta el asiento óseo tomando dos muestras una de la zona de la encía vestibular anterior superior y una de la zona de mucosa yugal zona posterior ambas incluyen bordes de la lesión y tejido circundante. Las muestras midieron aproximadamente 0,3 y $0,5 \mathrm{~mm}$ y fueron colocadas en una solución de formalina al 10\% acompañada por el protocolo de biopsia correspondiente para su análisis en el Servicio de Anatomía Patológica de la Facultad de Odontología de la UNNE. En el estudio histopatológico se observó dos fragmentos de mucosa uno de ellos con leve hiperqueratosis, acantopapilomatosis irregular, exocitosis y borramiento de la interfase del epitelio-corion, con degeneración vacuolar de las células basales y presencia de células epiteliales apoptóticas formando cuerpos eosinófilos densos (Cuerpos de Civatte), de localización intraepitelial y en el corion superficial. El fragmento restante mostró ulceración. A nivel del corion se reconoce extenso infiltrado inflamatorio mononuclear en banda, edema y congestión. Los extendidos citológicos realizados presentaban rica celularidad compuesta por células pavimentosas superficiales, intermedias y algunas escamas epiteliales, colgajos de células parabasales sobre un fondo inflamatorio rico en leucocitos polimorfonucleares. El diagnóstico definitivo fue de Liquen Rojo Plano Erosivo.

\section{Tratamiento}

En primer lugar se eliminaron los factores locales que pudieran exacerbar la lesión como la realización de un tratamiento gíngivo-periodontal realizándole tartrectomías y raspados gingivales, así como también se procedió a realizar el pulido de las piezas dentarias. Se le indicó realizar enjuagues con clorehexidina al 0,2 \% (sin alcohol) dos veces al día y cliotrimazol $0,25 \mathrm{mg}$ más triamcinolona $2,8 \%$, vitamina E $200000 \mathrm{UI}$ más vitamina A 125000 UI Lidocaíana $0,2 \%$ en orobase gel topicaciones 5 veces por día durante 7 días luego 15 días más la misma crema pero sin clotrimazol. Las topicaciones se realizaron con hisopo estéril en las áreas de la lesión. Se la derivo a su médico para controlar su stress.

\section{Discusión}

La paciente se encuentra ubicada dentro de la quinta década de la vida lo que coincide con el estudio realizado por Fang. M y colaboradores, en un estudio retrospectivo de 23 casos en el que la edad promedio oscilaba en los 52 años?. La lesión en este caso presenta dos lugares diferentes de ubicación en encía de maxilar superior y también en mucosa yugal, en zona de trígono retromolar y también así lo expresa Xue JL, Fan MW, Wang SZ, Chen XM, LiY, Wang en un estudio de 674 casos en donde cerca del $90,9 \%$ de los pacientes que tenían múltiples sitios en cavidad bucal 60 casos se presentaban en el labio superior $(8,9 \%)$ y en la encía un solo caso $(0,2 \%)^{10}$ y Guiglia R, Di Liberto C, Pizzo G" observaron la presencia de Liquen Plano en encía libre en 30 pacientes. En cuanto al sexo el presente caso clínico que corresponde al sexo femenino como en el Cortés- Ramírez D A, Gainza-Cirauqui ML en donde hubo predilecciónpor el género femenino y coincide con la etapa premenopáusica ${ }^{12}$. También el factor etiológico se asocia con el stress con lo expresado por Vergara Hernández C, Díaz Caballero A, Barrios García L, y también por Krasowska D, Pietrzak A, Surdacka A, Tuszy et. quienes su- 


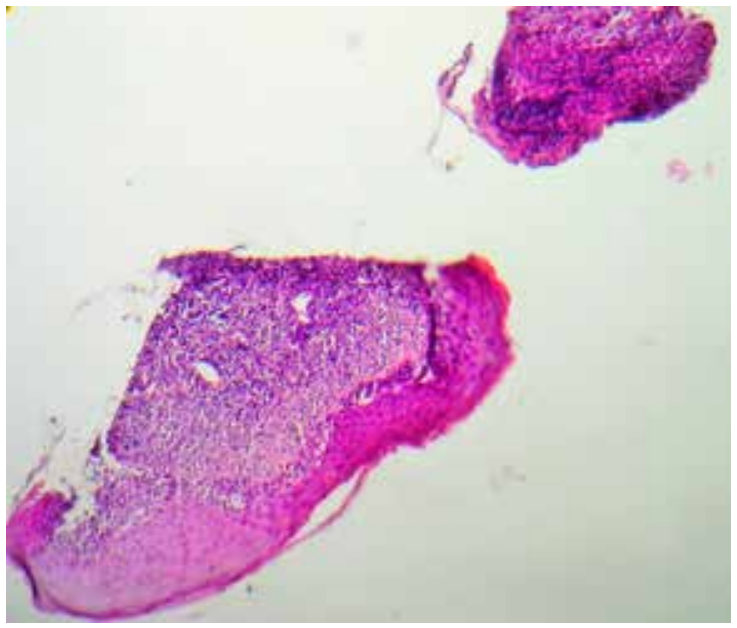

Foto $N^{\circ}$ I. I0 X H/E -Vista panorámica de lesión con hiperqueratosis, borramiento de la interfase epitelio- corion por intenso infiltrado inflamatorio mononuclear. El otro fragmento presenta pérdida del revestimiento epitelial.

gieren que el stress puede alterar numerosos parámetros de respuesta inmune y endocrina en el liquen plano que proporciona apoyo a su papel en la etiopatogenia de esta enfermedad ${ }^{\prime 2}$. Clínicamente la presentación de la lesión es del tipo erosivo coincidiendo con Mignogna MD, Lo Russo L, Fedele $S$ quienes reportan que una de las presentaciones más comunes son las del tipo erosivo ${ }^{13}$. Igualmente Bagán y Cerero dividen clínicamente al liquen en formas reticulares, correspondientes a las de predominio blanco y formas atróficoerosivas, correspondientes a las de predominio rojo. Bagán et alt., encuentran que las formas atrófico-erosivas predominan sobre las reticulares en una proporciónde 3 a $I$ aproximadamente ${ }^{14}$ confirmando los resultados de otros investigadoresy coincidiendo con nuestro caso clínico.En cuanto al cuadro histológico, encontramos en nuestro caso una ulceración por una solución de continuidad en el epitelio, con un cuadro de hiperqueratosis engrosamiento de la capa granular y degeneración hidrópico-vacuolar de la lámina basal con infiltración linfocitaria coincidiendo con lo encontrado por Vergara Hernández C.I Caballero A. et ${ }^{2}$. Haciendo referencia al tipo de tratamiento instaurado se coincide con Guiglia R, Diliberto C, Pizzo G, Picone $L$, quienes realizaron biopsia incisional de

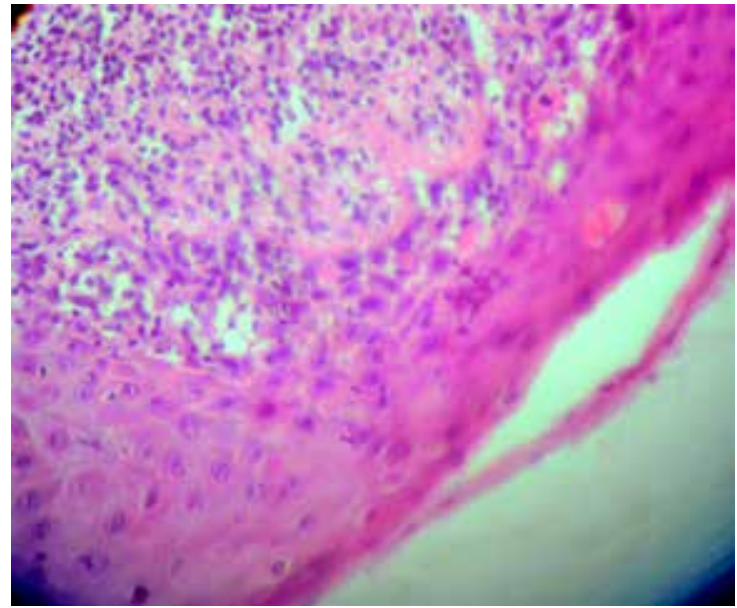

Foto $N^{\circ}$ 2. 40X H/E- Mayor detalle de la zona de daño basal, exocitosis, queratinocitos apoptóticos (Cuerpos de Civatte) e intenso infiltrado mononuclear que compromete el corion superficial y el estrato basal del epitelio.

una de las lesiones comprobando el diagnóstico de liquen plano para luego instaurar un protocolo combinado de higiene oral y la terapia de corticosteroides además de la eliminación del agente causal" El más utilizado es el gel de Orabase, porque brinda excelentes propiedades adhesivas y recomiendan que se aplique una dosis de cinco a seis veces al día de hidrocortisona. Ello coincide con el presente caso, en el cual el excipiente utilizado como vehículo fue el gel de Orabase y la frecuencia de la dosis empleada fue de cinco veces al día. Se obtuvieron resultados favorables con diez días de terapia ${ }^{15}$. 


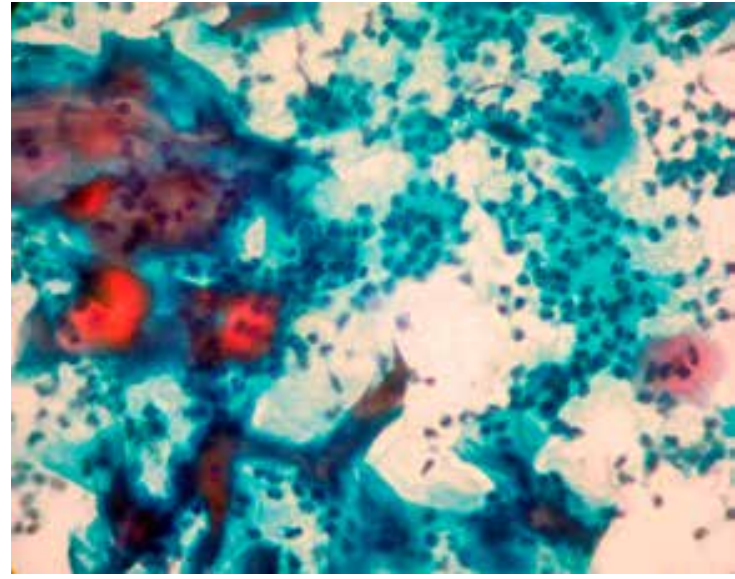

Foto $N^{\circ} 3$. Extendido citológico-PAP 40X- Extendido rico en células, pavimentosas superficiales e intermedias acompañadas por un fondo inflamatorio con leucocitos polimorfonucleares y linfocitos.
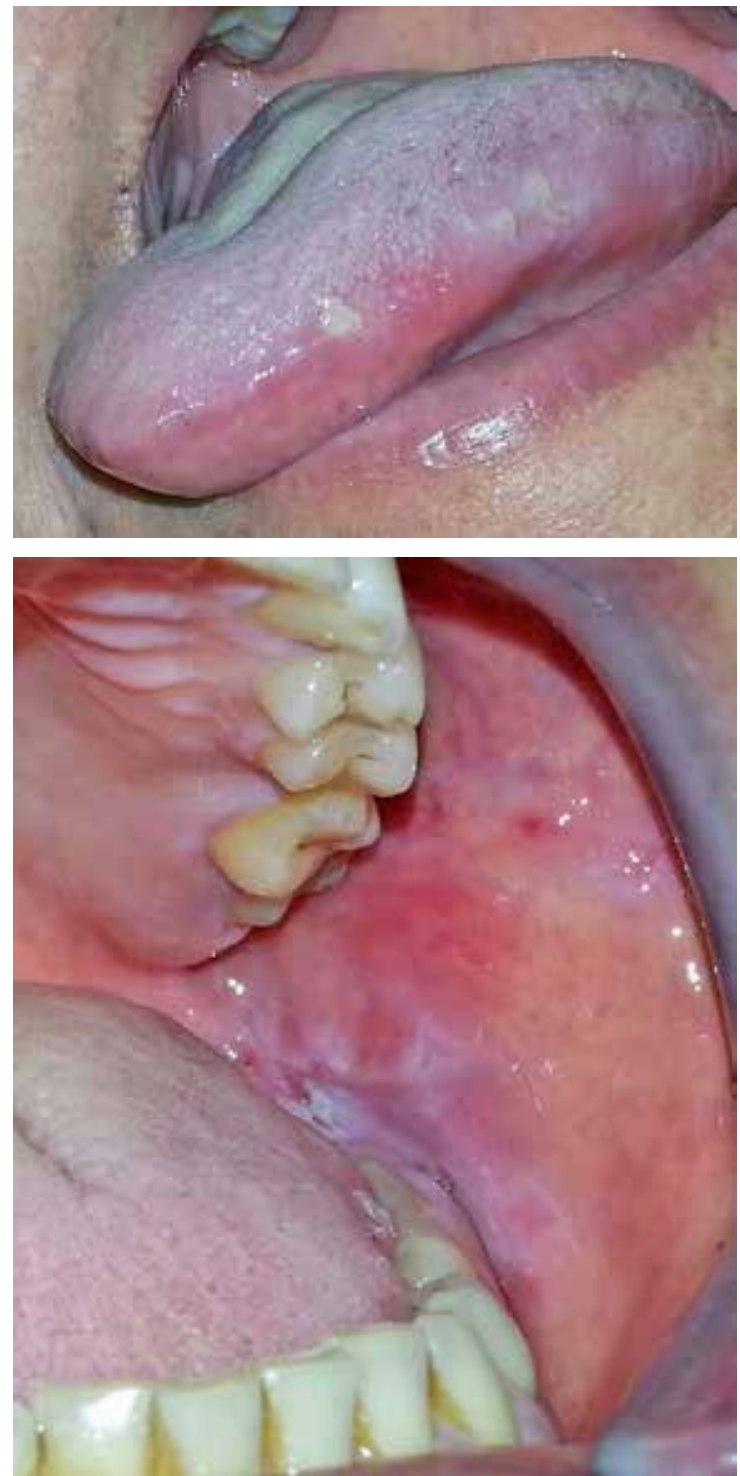

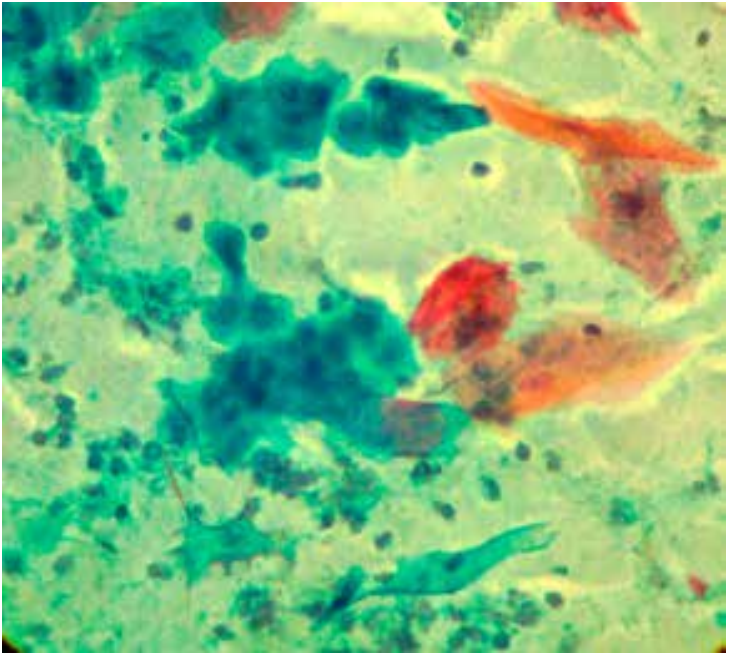

Foto $\mathbf{N}^{\circ}$ 4. Extendido citológico PAP 40X-células pavimentosas superficiales y escamas epiteliales.Se reconocen colgajos de células parabasales acompañadas por un fondo inflamatorio. 


\section{Bibliografía}

I. Rivadávio, Fernandes Batista de Amorim, João Luiz de Miranda, Manuel Antonio Gordón Nuñez, Ana Miryam Costa de Medeiros y Roseana de Almeida Freitas. Liquen Plano Oral con presentación Clínica Variada e Historia Familiar: Relato de un caso Volumen $42 \mathrm{~N}^{\circ} 2$.

2. Clara Inés Vergara Hernández, Antonio Díaz Caballero, Lía Barrios García-Liquen Plano en cavidad oral. Reporte de un caso clínico y revisión de la literatura - Acta Odontológica Venezolana Volumen $49 \mathrm{~N}^{\circ} 4$ / 201 I

3. Joseph A. Regezi- James Sciubba- Patología Bucal-3 ${ }^{a}$ Edición- Edit. Interamericana- Mc GrawHill- Pag. I 18-125

4. Bascones-llundain C, González Moles MA, Carrillo de Albornoz A, Bascones-Martínez. Liquen plano oral .Aspectos clínicos, etiopatogénicos y epidemiológicos- Av. Odontoestomatol. 2006; 22-I: II-I9.

5. Bermejo-Fenoll, Ambrosio y Lopez-Jornet, Pía. Liquen plano oral: Naturaleza, aspectos clínicos y tratamiento. RCOE [online]. 2004, vol.9, n.4 [citado 20I4-12-02], pp. 395-408.

6. Urbizo Vélez Joaquín. Liquen plano bucal y displasia epitelial. Rev.CubanaEstomatol. [Revista en la Internet]. 2013 Junio [citado 2014 Noviembre 27]; 50(2)

7. Cerero-Lapiedra, R..Malignización del liquen plano oral. Av Odontoestomatol [online]. 2008, vol.24, n-I

8. Garcia-Pola Vallejo, M.J. y García Martin, J.M. Tratamiento del liquen plano oral: una revisión. Av. Odontoestomatol [online]. 2008, vol.24, n.I
9. Fang M, Zhang W, Chen Y, He Z. Malignant transformation of oral lichen planus: a retrospective study of 23 cases. Quintessence Int. 2009 Mar; 40(3):235-42

10. Xue JL, Fan MW, Wang SZ, Chen XM, Li Y, Wang L. A clinical study of 674 patients with oral lichen planus in China. J Oral Pathos Med. 2005 Sep; 34(8):467-72.

II. Guiglia R, Di Liberto C, Pizzo G, Picone L, Lo Muzio L, Gallo PD, Campisi G, D'Angelo M. A combined treatment regimen for desquamative gingivitis in patients with oral lichen planus.J Oral Pathol Med. 2007 Feb; 36(2): I 10-6.

12. Cortés-Ramírez DA, Gainza-Cirauqui ML, Echebarria-Goikouria MA, Aguirre-Urizar JM.Oral lichenoid disease as a premalignant condition: the controversies and the unknown. Med Oral. Patol. Oral Cir Bucal. 2009 Mar I; I4 (3):EI I822.

13. Mignogna MD, Lo Russo L, Fedele S. Gingival involvement of oral lichen planus in a series of 700 patients. J Clin.Periodontol. 2005 Oct.; 32(10): 1029-33.

14. Bagán JV, Cerero R. Liquen plano oral. En: Bagán JV, Ceballos A, Bermejo A, Aguirre JM, Peñarrocha M. Medicina oral. Barcelona: Masson, S.A., 1995; 202-19.

15. Harris J, Díaz-Caballero A, Fortich N. Manejo estomatológico del liquen plano oral atrófico. Revisión de literatura y presentación de un caso. Univ. Odontol. 20I I Jul-Dic; 30 (65): I05-I I0. 